\title{
Minimizing Time Delay of Information Routed Across Dynamic Temporal Sensor Networks
}

\author{
Michael J. Hirsch $]^{1}$, Azar Sadeghnejad ${ }^{2}$ \\ ${ }^{1}$ ISEA TEK, LLC, 32751, U.S.A. \\ ${ }^{2}$ Radiation-Oncology Department, University of Texas - Southwestern Medical Center, 75390, U.S.A.
}

A R T I C L E IN F O

Article history:

Received: 13 July, 2018

Accepted: 10 August, 2018

Online: 20 August, 2018

\section{Keywords:}

Information Routing

Sensor Network

Communication Topology

\begin{abstract}
A B S T R A C T
In this research we address the problem of routing information across dynamic temporal sensor networks. The goal is to determine which information, generated by sensors on resources at various times, is able to be routed to other resources, consumer resources, within the given information time window, while being constrained by temporally dynamic bandwidth limitations across the sensor network, and storage limitations on the resources. A mathematical model of the problem is derived, and used to find solutions to the problem. In addition, a heuristic is developed to efficiently find good quality solutions. Monte-Carlo simulations are performed comparing solutions found by commercial software with the heuristic.
\end{abstract}

\section{Introduction}

This paper is an extension of work originally presented at the Military Communications Conference [1]. Wireless sensor networks are a class of networks in which some or all of the sensors or resources collect, analyze, and communicate data acquired from their environment (external or internal) to other nodes in the network [2]. These other nodes could be resources, sensors, or fixed or mobile command posts. Mobile adhoc networks (MANETs) are a subset of wireless sensor networks that have an absence of a fixed infrastructure, and exhibit a dynamic communication topology [3]. Resources within transmission range of others communicate directly (depending on bandwidth limitations) and cooperation among intermediate resources is required for resources to communicate with others outside of their direct communication range. More and more networks, in both the military and commercial domain, are relying on wireless sensor networks and MANETs [4, 5, 6].

The ability for data to be collected by manned and unmanned vehicles engaged in military, government, and commercial missions is increasing at an exponential rate [7]. Examples include high-resolution video, telephone intercepts, traffic congestion reports, internal vehicle system states, among others. In commercial telecommunication networks, there are instances where the capacity across the network is in the ter- abytes per second range [8]. Networks of this type have the ability to transmit all data that is collected without restrictions or delays. However, in times of crisis and disaster, commercial and civil communication networks can be severely degraded [9, 10]. And in military missions, bandwidth is oftentimes severely limited, which results in a reduction in transmission and sharing of information that can be mission-critical. And this will continue to be the case due to the rise of operations performed in anti-access/area denial (A2AD) environments, against near-peer adversaries. In the past, this meant that information collected by vehicles would not be processed, analyzed, and understood until after the vehicle had returned to base and 'uploaded' all of the collections [7, 11].

In performing more of the processing and analysis on-board the vehicles, there has the potential for less bandwidth requirements for vehicles to share information important for mission success. In effect, this can be seen as pushing the smarts out to the tactical edge. Each generation of manned and unmanned vehicles is having more and more processing capabilities onboard [11]. Examples include internally monitoring on-board systems, fusing information collected from various on-board sensors, picking out a person of interest from a full motion video, and deciding how much information should be sent over the sensor network and to whom should be the recipient of such information [12]. While some of the collected information can

${ }^{*}$ Michael J. Hirsch, ISEA TEK, 620 N. Wymore Rd., Ste. 260, Maitland, FL 32751 U.S.A., mhirsch@iseatek.com 
just be generated and stored on a vehicle, and offloaded upon mission completion, other generated information is vital to mission success, and must be transmitted to control station(s) or other resources during mission execution.

To address this, we consider the following problem. There are a set of resources (moving or fixed) that have a set of Information Generation Requests (IGRs) to satisfy over a given time horizon. If a resource executes a task to perform the IGR, then an associated piece of information is generated. We will use the term IGR to denote the information that gets generated. Each IGR can come from one of a fixed set of categories (for an example of information categories, see Table 1). In addition, each IGR has associated with it the following characteristics: an expected time of generation, the set of consumer resources (those resources that would find this information important for their mission), the time by which the information needs to be delivered to the to be of use, the expected original size of the information, and the priority of the information (i.e., a measure of its importance in the mission). We note that the priority of an IGR could be determined a priori (e.g., information collected about one kinematic location is deemed very important and therefore is given a high priority) or dynamically on-board the resource at the time of collection, if the resource has the appropriate analytics (e.g., image is given low priority because it does not contain any red cars). By a 'consumer resource' for a particular IGR, we mean that the resource actually performs some processing with that information (e.g., fuses that information with other information [13, 14], makes command and control decisions, etc.).

There is a dynamic sensor network topology in place over the time horizon. This dynamic network has finite, but varying bandwidth limitations between any two resources at any given time, and in many cases there might not be a direct connection between two resources. Each IGR can be sent over the network with different potential granularities (e.g., 100\% of original size, $70 \%$ of original size, etc.), but there is a minimum size percentage with which the information remains useful to the consumer of the information. As an IGR gets routed from the generating resource to the consumer resource, all resources along this route will need to store this IGR locally for some amount of time. The generating resource will need to store an IGR from the time of generation until the IGR is completely sent to the next resource in the path to the consumer resource(s). Non-consumer resources along this path will need to store the IGR from the time the resource begins receiving the IGR until the time the resource has completely transmitted the IGR to the next resource along the path. And the consumer resource(s) will need to store the IGR form the time they begin receiving it until at least they have finished processing the IGR.

Each resource has a finite storage capacity, and resources can triage information they have stored (e.g., once a piece of information is generated and completely sent to the next resource along the path to the consumer, the generator resource can delete the information from storage). The objective is then to determine which IGRs to route over the network, how to route those IGRs so that they have a minimal time delay (difference between time of consumption and time of generation), from generating resource to consumer resource over the time horizon, while also attempting to maximize the size of granularity of each IGR that is routed. In turn, this produces the higher-level end product of determining which IGRs should be accomplished for mission objectives, given the network topology characteristics.

There has been some prior research into routing schemes for collected data across a dynamic network. In [17], the author considered the problem of sinkholes in a network. A sinkhole node attempts to deceive all the nodes in the network to route network traffic to the sinkhole, by broadcasting false routing information across the network. An approach based upon secondary caching was developed to prevent a sinkhole attack in dynamic source routing sensor networks. In [18], the author consider cellular data networks and the significant increase in data being transmitted over these networks. Their research looked at data traffic management techniques, whereby users and automated approaches 'flag' messages that are of low priority, and an higher-level agent-based scheduling system had the ability to delay scheduling of low priority messages during peak load times. In [19], the authors considered the problem of extending lifetimes of wireless sensor networks. In their view, the sending of redundant data across sensor networks reduced the lifetime of these networks. They looked at providing certain nodes in the network to function as 'data aggregation' nodes, receiving collected data from other nodes in the network, fusing or aggregating the data together to reduce redundant information, and then passing this reduced set of data onwards to a base station. They employed a grid-based routing and aggregator selection scheme to find the minimum number of aggregation points while routing data to the base station ensuring that the network lifetime is maximized.

In addition, there has been some prior research addressing certain aspects of our problem of interest, but none that has addressed the problem completely. In [20], the author considered the problem of routing information across a network, from a workflow perspective, but the network was assumed to be complete and static, and bandwidth was not addressed. In [21], the researchers extended this work to include bandwidth limitations, but still under the assumption on a complete and static network. In [22], the researchers presented a mixed-integer linear programming formulation to model the trajectory of a set of unmanned aerial vehicles, along with routing of information collected from these vehicles back to a base station. They did not consider bandwidth fluctuations in the sensor network, and the resources were not the recipient of any information, just the base station. In addition, all of their information was considered to have the same priority. In [23], the author considered underwater 
Table 1: Examples of Information Categories.

\begin{tabular}{l|l} 
Category & Example \\
\hline Internal System State & Measurements on the engine pressure ratio [15] \\
External Collection & Photographs of an urban traffic intersection \\
Situation Assessments & High likelihood that red truck contains person of interest \\
Command and Control (C2) & Modifying flight plan to conserve battery life [16] \\
\hline
\end{tabular}

acoustic networks and the long propagation delays and low bandwidths inherent in such networks. A set of nodes was using the same bandwidth channel, and because their problem had bursty data, the solution approach looked at submitting their requests randomly, as demand dictated. A medium access control protocol was in place to minimize the number of collisions among the information flowing across the network. In [24], the researchers investigated ways to enhance the operations of a power grid. One of the ways, taking advantage of the high sampling rates of the measurement data, required a high bandwidth, networked communication system. Their results derived a method to simulate, design, and test the adequacy of a communication system for a particular grid layout.

The rest of this paper is organized as follows. In Section 2, we introduce the parameters and decision variables for our model, and rigorously derive the mathematical program representing our problem. Section 3 develops a heuristic to efficiently find good quality solutions. Section 4 analyzes the results of computational experiments performed on various scenarios, comparing commercial software solving a linearized version of the mathematical program and the solutions found by the heuristic. Section 5 provides some concluding remarks and future research directions.

\section{Mathematical Formulation}

In this section, we rigorously derive mathematical equations to model the problem of interest. This results in a mixed-integer nonlinear program (MINLP). We note that the time dimension in the model is discretized, over the planning horizon of $\mathcal{K}$ time-steps.

\subsection{Parameters}

This section lists the parameters for the mathematical programming problem.

$\mathcal{I}$ is the set of resources, $i, i_{1}, i_{2}, \in \mathcal{I}$;

$\mathcal{J}$ is the number of Information Generation $\mathrm{Re}$ quests (IGR)s, $j \in\{1, \ldots, \mathcal{J}\}$;

$\mathcal{K}$ is the number of time-steps in the planning horizon, $k, \hat{k} \in\{1, \ldots, \mathcal{K}\}$;

$\mathcal{P}^{\text {min }}$ is the minimum percentage an IGR can be decreased in size and still be considered useful to consumer resource(s);

$\tau_{i, j}$ is the number of time-steps after a consumer receives an IGR before the consumer can delete the IGR, i.e., the number of time-steps it takes resource $i$ (the consumer resource) to 'process' IGR $j$;

$\rho_{j}$ is the expected priority of IGR $j$;

$S_{j}^{o}$ is the expected original size of IGR $j$;

$g_{j}$ is the expected time of generation of IGR $j$;

$d_{j}$ is the time by which IGR $j$ is due to the consumer resource(s) of the IGR;

$G_{i, j}= \begin{cases}1 & \text { if resource } i \text { is tasked with generating IGR } j \\ 0 & \text { o.w. }\end{cases}$

$C_{i, j}=\left\{\begin{array}{ll}1 & \text { if resource } i \text { is a consumer of IGR } j \\ 0 & \text { o.w. }\end{array} ;\right.$

$L_{i}$ is the storage capacity of resource $i$;

$b_{i_{1}, i_{2}, k}$ is the expected bandwidth capacity from resource $i_{1}$ to resource $i_{2}$ at time-step $k$;

$\bar{\rho}$ is the largest expected priority, i.e., $\bar{\rho}=$ $\max _{j}\left[\rho_{j}\right]$;

$\omega_{d}$ and $\omega_{s}$ are the weighting coefficients for the priority and size components of the objective function;

$\mathcal{M}$ is a large enough constant, used in Constraints (7).

\subsection{Variables}

This section lists the decision variables for the mathematical programming problem.

$$
x_{j}=\left\{\begin{array}{l}
1 \quad \begin{array}{l}
\text { if IGR } j \text { is chosen to be routed } \\
\text { from the generating resource to } \\
\text { the consumer resource (s) }
\end{array}, \forall j ; \\
0 \quad \text { o.w. }
\end{array}, \forall\right.
$$

$s_{j}=$ the size of IGR $j$ sent to the consumer resource(s), $\forall j$;

$a_{i_{1}, i_{2}, j, k}=$ the amount (size) of IGR $j$ sent from resource $i_{1}$ to resource $i_{2}$ during time-step $k, \forall i_{1}, i_{2}\left(\neq i_{1}\right), j, k$;

$$
\begin{aligned}
& \alpha_{i_{1}, i_{2}, j, k}= \begin{cases}1 & \text { if resource } i_{1} \text { sends some of IGR } j \\
\text { to resource } i_{2} \text { during time-step } k & \text { o.w. }\end{cases} \\
& \forall i_{1}, i_{2}\left(\neq i_{1}\right), j, k ;
\end{aligned}
$$


$m_{i, j, k}=$ the amount of IGR $j$ being stored on resource $i$ at time-step $k, \forall i, j, k$. N.B.: For consistency of the constraints, $m_{i, j, 0}=0 \forall i, j$;

$\phi_{i_{1}, i_{2}, j}=\left\{\begin{array}{ll}1 & \text { if resource } i_{1} \text { is assigned to send } \\ & \text { IGR } j \text { to resource } i_{2} \\ 0 & \text { o.w. }\end{array}\right.$,

$$
\forall i_{1}, i_{2}\left(\neq i_{1}\right), j
$$

$\beta_{i, j}=$ the amount of time resource $i$ takes in sending IGR $j$ to other resources, i.e., the time between resource $i$ completely receiving or generating IGR $j$ to the time where resource $i$ no longer needs IGR $j, \forall i, j$;

$t_{i, j, k}=\left\{\begin{array}{l}1 \quad \begin{array}{l}\text { if resource } i \text { cumulatively receives } \\ \text { (or generates) } s_{j} \text { size units of IGR } j \text { at } \\ \text { time-step } k\end{array} \\ 0 \quad \text { o.w. }\end{array}\right.$

$$
\forall i, j, k \text {; }
$$

$T_{i, j}=$ the time-step at which resource $i$ completely receives (or generates) IGR $j, \forall i, j$;

$d_{i, j, k}=\left\{\begin{array}{ll}1 & \text { if resource } i \text { deletes IGR } j \text { from } \\ & \text { storage at time-step } k \\ 0 & \text { o.w. }\end{array}\right.$,

$$
\forall i, j, k \text {; }
$$

$D_{i, j}=$ the time-step at which resource $i$ deletes IGR $j$ from its storage, $\forall i, j$;

$Q_{i, j}=$ the time at which resource $i$ completely consumes (or transmits) IGR $j, \forall i, j$;

$e_{i, j, k}=\left\{\begin{array}{ll}1 & \text { if resource } i \text { receives (or generates) } \\ & \text { IGR } j \text { by time-step } k \\ 0 & \text { o.w. }\end{array}\right.$,

$$
\forall i, j, k \text {. }
$$

N.B.: For consistency of the constraints, it is necessary to set $e_{i, j, 0}=0 \forall i, j$. Also, note that $e_{i, j, k}=1$ implies that $e_{i, j, \ell}=1$ for each $\ell>k$ and $e_{i, j, k}=0$ implies that $e_{i, j, \ell}=0$ for each $\ell<k$.

\subsection{Nonlinear Mathematical Formulation}

This section presents the mathematical model of the problem described in Section 1, resulting in a mixedinteger nonlinear program (MINLP).

$$
\begin{aligned}
\max \omega_{d} \cdot\left(\sum_{j=1} \sum_{\substack{i \in \mathcal{I}: \\
C_{i, j}=1}} \frac{\left(\mathcal{K}+g_{j}\right) \cdot x_{j}-T_{i, j}}{\mathcal{K}}\right) \\
+\omega_{s} \cdot\left(\sum_{j=1}^{\mathcal{J}} \frac{s_{j}}{S_{j}^{o}}\right)
\end{aligned}
$$

s.t.

$$
s_{j} \leq S_{j}^{o} \cdot x_{j} \forall j
$$$$
s_{j} \geq \mathcal{P}^{\min } \cdot S_{j}^{o} \cdot x_{j} \forall j
$$

$\sum_{j} m_{i, j, k} \leq L_{i} \forall i, k$

$m_{i, j, k}=m_{i, j, k-1}+\sum_{\substack{i_{1} \in \mathcal{I} \\ i_{i} \neq i}} a_{i_{1}, i, j, k}$

$$
-d_{i, j, k} \cdot s_{j}+\delta\left[g_{j}-k\right] \cdot G_{i, j} \cdot s_{j} \forall i, j, k
$$

$\sum_{k=1}^{\mathcal{K}} \alpha_{i_{1}, i_{2}, j, k} \leq \mathcal{K} \cdot x_{j} \forall i_{1}, i_{2}, j$

$\mathcal{M} \cdot \alpha_{i_{1}, i_{2}, j, k} \geq a_{i_{1}, i_{2}, j, k} \quad \forall i_{1}, i_{2}, j, k$

$\sum_{j} a_{i_{1}, i_{2}, j, k} \leq b_{i_{1}, i_{2}, k} \quad \forall i_{1}, i_{2}, k$

$\sum_{k=1}^{g_{j}} a_{i_{1}, i_{2}, j, k}=0 \quad \forall i_{1}, i_{2}, j$

$\sum_{k=1}^{\mathcal{K}} a_{i_{1}, i_{2}, j, k} \leq s_{j} \forall i_{1}, i_{2}, j$

$$
\begin{aligned}
e_{i, j, k} & >\sum_{\substack{i_{1} \in \mathcal{I} \\
i_{1} \neq i}} \sum_{\hat{k}=1}^{k} a_{i_{1}, i, j, \hat{k}} \\
& -s_{j}-\left(1-x_{j}\right) \quad \forall k, \forall i, j \text { s.t. } G_{i, j}=0
\end{aligned}
$$

$e_{i, j, k} \cdot s_{j}$

$$
\leq \sum_{\substack{i_{1} \in \mathcal{I} \\ i_{1} \neq i}} \sum_{\hat{k}=1}^{k} a_{i_{1}, i, j, \hat{k}} \forall k, \forall i, j \text { s.t. } G_{i, j}=0
$$

$t_{i, j, k} \geq e_{i, j, k}-e_{i, j, k-1} \quad \forall i, j, k$

$\sum_{k=1}^{\mathcal{K}} t_{i, j, k} \leq x_{j} \quad \forall i, j$

$t_{i, j, g_{j}}=x_{j} \forall i, j$ s.t. $G_{i, j}=1$

$T_{i, j}=\sum_{k=1}^{\mathcal{K}} k \cdot t_{i, j, k} \quad \forall i, j$

$T_{i, j} \leq d_{j} \cdot x_{j} \forall i, j$

$Q_{i, j}=T_{i, j}+\tau_{i, j} \cdot x_{j} \forall i, j$ s.t. $C_{i, j}=1$

$Q_{i, j}=T_{i, j}+\beta_{i, j} \forall i, j$ s.t. $C_{i, j}=0$

$\beta_{i_{1}, j} \geq k \cdot \alpha_{i_{1}, i_{2}, j, k}-T_{i_{1}, j} \forall i_{1}, i_{2}, j, k$

$D_{i, j}>Q_{i, j}-\left(1-\sum_{k=1}^{\mathcal{K}} t_{i, j, k}\right) \forall i, j$ 


$$
\begin{aligned}
& D_{i, j}=\sum_{k=1}^{\mathcal{K}} k \cdot d_{i, j, k} \forall i, j \\
& \sum_{k=1}^{\mathcal{K}} d_{i, j, k} \leq x_{j} \forall i, j \\
& \sum_{\substack{i_{1} \in \mathcal{I} \\
i_{1} \neq i}} \phi_{i_{1}, i, j} \leq x_{j} \quad \forall i, j \\
& \sum_{k=1}^{\mathcal{K}} \alpha_{i_{1}, i_{2}, j, k} \leq \mathcal{K} \cdot \phi_{i_{1}, i_{2}, j} \forall i_{1}, i_{2}, j \\
& \sum_{k=1}^{\mathcal{K}} \alpha_{i_{1}, i_{2}, j, k} \geq \phi_{i_{1}, i_{2}, j} \forall i_{1}, i_{2}, j \\
& \sum_{\substack{i_{2} \in \mathcal{I} \\
i_{2} \neq i}} \alpha_{i, i_{2}, j, k} \leq \sum_{\hat{k}=1}^{k-1} t_{i, j, \hat{k}} \forall i, j, k \\
& s_{j}=\sum_{k=1}^{\mathcal{K}} \sum_{\substack{i_{1} \in \mathcal{I} \\
i_{1} \neq c_{j}}} a_{i_{1}, c_{j}, j, k} \forall j \\
& \sum_{k=1}^{\mathcal{K}} \sum_{i_{2} \in \mathcal{I}} \alpha_{c_{j}, i_{2}, j, k}=0 \quad \forall j \\
& x_{j}, \alpha_{i_{1}, i_{2}, j, k}, \phi_{i_{1}, i_{2}, j}, t_{i, j, k}, d_{i, j, k}, e_{i, j, k} \in\{0,1\} \\
& \forall i, i_{1}, i_{2}, j, k \\
& s_{j}, a_{i_{1}, i_{2}, j, k}, m_{i, j, k} \in\left[0, S_{j}^{o}\right] \forall i, i_{1}, i_{2}, j, k \\
& \beta_{i, j}, D_{i, j}, T_{i, j}, Q_{i, j} \in[0, \mathcal{K}] \cap \mathbb{Z} \forall i, j, k
\end{aligned}
$$

\subsection{Interpretation of Nonlinear Mathe- matical Formulation}

In this section, we explain the objective function and each of the constraints that are part of the mathematical formulation.

- The objective function, Equation (1), is a weighted combination of the time delay and size of the IGRs routed across the network.

The first term in the objective function deals with the time delay of those IGRs chosen to be routed. The sum is over all IGRs $j$ and all resources $i$ that are consumers of IGR $j$ (i.e., those resources such that the parameter $\left.C_{i, j}=1\right)$. When an IGR $j$ is not chosen to be routed, $x_{j}$ is set to be 0 and $T_{i, j}$ also ends up as 0 , so this term is 0 . When an IGR is chosen to be routed, $x_{j}$ is set to be 1 and $T_{i, j}$ is set to be the time at which resource $i$ completely receives IGR $j$. The time IGR $j$ is generated is given by the parameter $g_{j}$. The maximum value of this term is 1 , which occurs when $T_{i, j}=g_{j}$ (which is an idealistic situation), while the minimum value of this term is $\frac{\mathcal{K}+g_{j}-d_{j}}{\mathcal{K}}$, which can be 0 when $g_{j}$ is 0 and $d_{j}$ is $\mathcal{K}$.

The second term of the objective function deals with the size of the IGRs chosen to be routed, looking at the ratio of the actual size of the IGR that gets routed versus the original size.

Note that that scale of these two terms are the same, i.e., they are both within $[0,1]$, and are unit-less. The first term is weighted by $\omega_{d}$, while the second term is weighted by $\omega_{s}$.

- Constraints 22 force $s_{j}$ to be less than or equal to $S_{j}^{o}$ if IGR $j$ is chosen to be routed, and 0 if IGR $j$ is not chosen to be routed;

- Constraints 3 require $s_{j}$ to be greater than or equal to the minimal amount to be routed for IGR $j\left(\mathcal{P}^{\text {min }} \cdot S_{j}^{o}\right)$, if IGR $j$ is chosen to be routed;

- Constraints 4 ensure that at each time-step, the storage capacity for each resource is not exceeded;

- Constraints (5) determine the amount of IGR $j$ stored on resource $i$ at time $k$ as the amount stored at time $k-1$ plus the amount sent to resource $i$ at time-step $k$ minus $s_{j}$ if IGR $j$ is deleted from resource $i$ 's storage at time-step $k$, plus $s_{j}$ if IGR $j$ is chosen to be routed by resource $i$ and is generated at time-step $k$. N.B.: The Dirac-Delta impulse function $\delta[q]$ returns 1 when $q=0$ and returns 0 otherwise [25];

- Constraints (6) only allow $\alpha_{i_{1}, i_{2}, j, k}$ to be greater than 0 if IGR $j$ is chosen to be routed;

- Constraints 77 force $a_{i_{1}, i_{2}, j, k}$ to be 0 if resource $i_{1}$ is not sending IGR $j$ to resource $i_{2}$ at time-step $k$;

- Constraints 8 limit the amount of information traveling from resource $i_{1}$ to $i_{2}$ at time-step $k$ to be no more that the capacity of this edge, $b_{i_{1}, i_{2}, k}$;

- Constraints 9 enforce that no resource can send IGR $j$ before it is generated;

- Constraints 10 enforce that resource $i_{1}$ send no more than $s_{j}$ of IGR $j$ to resource $i_{2}$;

- For those resources that do not generate IGR $j$ and IGR $j$ is chosen to be routed, Constraints 111 force $e_{i, j, k}$ to be 1 if all $s_{j}$ of IGR $j$ reaches resource $i$ at or before time-step $k$. This constraint allows $e_{i, j, k}$ to be 0 or 1 otherwise;

- For those resources that do not generate IGR $j$, Constraints 12 force $e_{i, j, k}$ to be 0 while not all $s_{j}$ of IGR $j$ has reached resource $i$. This constraint allows $e_{i, j, k}$ to be 0 or 1 otherwise;

- Constraints 13 set $t_{i, j, k}$ to be 1 at the time-step that resource $i$ receives all $s_{j}$ of IGR $j$, and to be 0 for all other time-steps;

- Constraints 14 enforce that resource $i$ can receive IGR $j$ at most once;

- Constraints 15 set $t_{i, j, k}$ equal to 1 if IGR $j$ is chosen to be routed, is generated by resource $i$, and $k$ is the time of generation of IGR $j$; 
- Constraints 16 set $T_{i, j}$ to be the time at which resource $i$ receives IGR $j$;

- Constraints 17 enforce that any resource that receives IGR $j$, must do so before its due date/time, if IGR $j$ is chosen to be routed;

- Constraints 18 define the time at which resource $i$ has completely 'processed' IGR $j$, if resource $i$ is a consumer of IGR $j$ and IGR $j$ is chosen to be routed;

- Constraints 19 define the time at which resource $i$ has completely transmitted IGR $j$ to other resources, if resource $i$ is not a consumer of IGR $j$ and IGR $j$ is chosen to be routed;

- Constraints 20 determine the amount of time it takes resource $i$ to send IGR $j$ to other resources, once resource $i$ receives or generates IGR $j$;

- Constraints 21 ensure that if IGR $j$ is chosen to be routed, that IGR $j$ cannot be deleted from resource $i$ until resource $i$ is finished with the IGR;

- Constraints 22 determine the time at which resource $i$ deletes IGR $j$;

- Constraints 23 ensure that resource $i$ can delete IGR $j$ at most once;

- Constraints 24 enforce that at most one resource is assigned to send IGR $j$ to resource $i$;

- Constraints 25 only allow resource $i_{1}$ to send IGR $j$ to resource $i_{2}$ if it is assigned to;

- Constraints 26 force $i_{1}$ to send IGR $j$ to resource $i_{2}$ during at least one time-step, if $i_{1}$ is assigned to send IGR $j$ to resource $i_{2}$;

- Constraints 27 does not allow resource $i$ to send any portion of IGR $j$ at time-step $k$ if resource $i$ has not completely received (or generated) IGR $j$ by time-step $k-1$;

- Constraints 28 ensure that the consumer resource(s) of IGR $j$ receives all $s_{j}$ units, if IGR $j$ is chosen to be routed;

- Constraints 29 prohibit resource $c_{j}$ (the consumer of IGR $j$ ) from sending IGR $j$ to other resources;

- Constraints 30 - 32 are domain restriction constraints on the decision variables.

\section{Solution Methodologies}

The formulation derived in Section 2.3 is a nonlinear mixed-integer programming problem. However, this formulation can be linearized through standard techniques from operations research [26], resulting in a mixed-integer linear program (MILP). As such, in theory the MILP formulation could be solved using a number of commercial software packages (e.g., CPLEX [27], LIND0 [28], etc.). It can be shown that this optimization problem is NP-Hard, as a variant of the vehicle routing problem with split deliveries and time windows [29, 30, 31], which necessitate heuristic strategies for finding good-quality solutions efficiently [32, 33] when the problem instances are of sufficient size.

The heuristic developed is split into two phases, an Information Routing phase, and a Storage Management phase. The Information Routing phase attempts to route all of the IGRs, from generating resource to consuming resource(s), without consideration of storage limitations on the individual resources. The only constraints taken into account in the Information Routing phase are the bandwidth limitations across the sensor network. The Storage Management phase of the heuristic then factors in the storage limitations of each resource to reduce the solution to one that is feasible.

Figures 2 and 1 provide pseudo-code for the Information Routing and Storage Management phases, respectively. The Information Routing function is input the set of IGRs (and all information about the IGRs), the initial allocation of bandwidth across the network over time (InitCommBW), the minimum percentage (in size) an IGR is able to be reduced and still be useful (MinSizePercent), and the ratio by which to reduce the size of the IGR in sending over the network, if needed (GranularityRatio). Lines 1 to 5 initialize internal variables. Lines 6 to 32 get executed while there are still IGRs on the list to be routed. For each IGR still on the CurListIGRs list, lines 7 to 25 attempt to determine a route for the IGR that allows for at least the minimum size for the IGR to be routed. Line $8 \mathrm{com}-$ putes the size of the current IGR and line 9 computes the minimal allowable size of the IGR to be routed. While a feasible route for the current IGR has not been found, and the current size of the IGR is larger then the minimum allowable size, lines 11 to 19 get executed. Line 11 calls the function ComputeRoute, given the current size of the IGR and the current allocation of bandwidth across the network. Returned from this function are the route for this IGR from generating resource to consumer resource, as well as the time the IGR will completely reach the consumer resource. If the time by which the consumer completely receives the IGR is less than the due date of the IGR, then a potential route for this IGR is found, and the potential time delay for this IGR is computed. Otherwise, the size of the IGR is reduced by the granularity ratio. If a potential route is not found in lines 11 to 19 , then this IGR is moved to the NotRoutedIGRs list and removed from the current list of IGRs (lines 21 and 22). Line 23 also sets this IGR to have a delay time of $+\infty$. After the code in lines 7 to 25 is completed, the heuristic greedily selects the IGR with the current minimal potential delay time (line 26). This IGR is removed from the list of current IGRs, and added to the list of routed IGRs. The potential route computed for this IGR is 


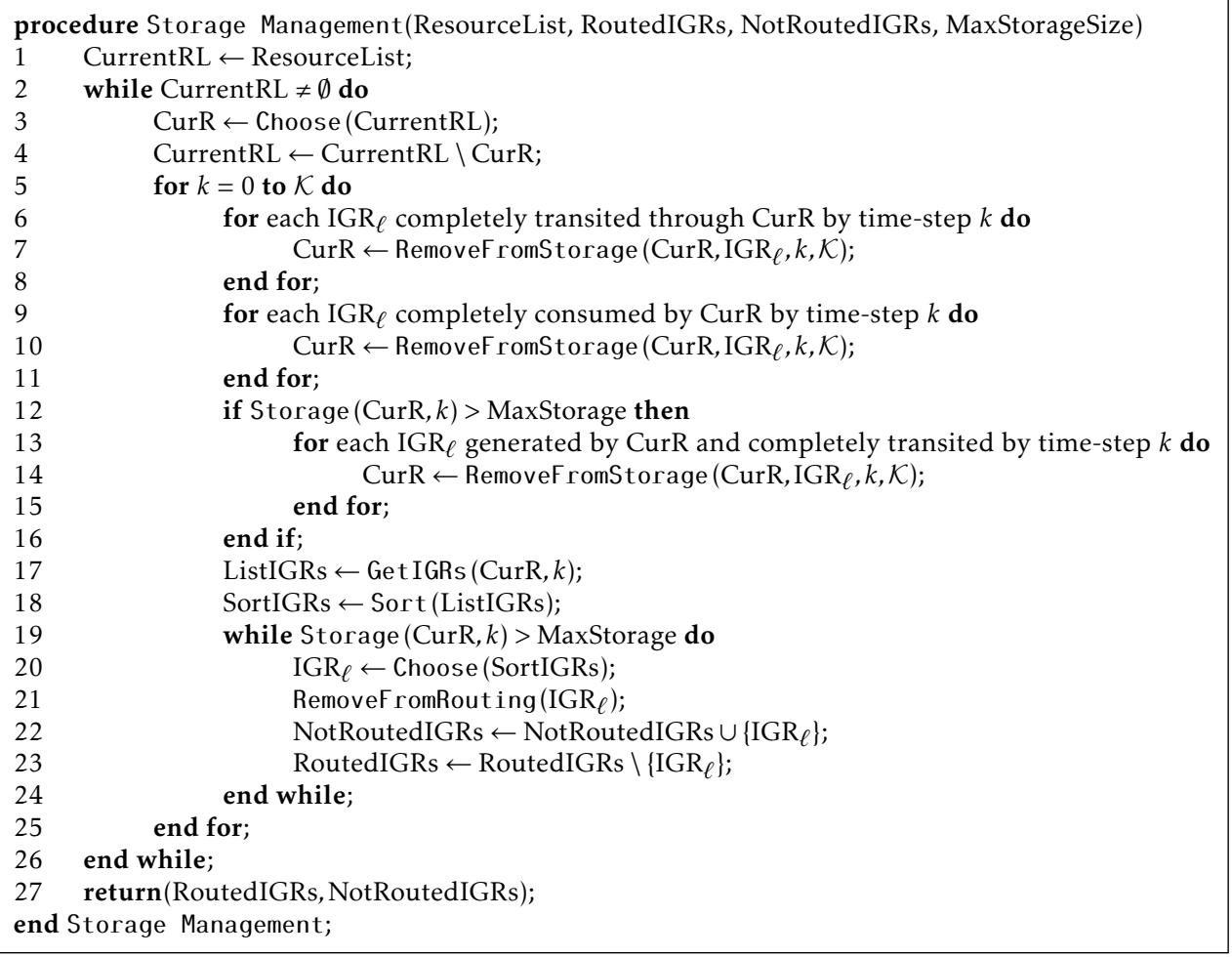

Figure 1: Pseudo-code for the Storage Management phase of the heuristic.

Table 2: Categories, and values used for the number of resources and density over the numerical experiments.

\begin{tabular}{c|c|c} 
Category & $\mathrm{N}$ & Density \\
\hline Extra Small & 3 & \\
Small & $4,5,6$ & $1,0.67,0.33$ \\
Medium & $8,10,12$ & \\
Large & $15,20,25$ & \\
\hline
\end{tabular}

saved into the set IGRRoutes in line 29, and the current bandwidth allocation across the temporal network is updated (due to this IGR having its route finalized) in line 30. This process in lines 6 through 32 continues until the set CurListIGRs is empty. The output from the Information Routing function is the set of IGRs that have been routed (along with all of the necessary details of the routes), as well as the set of IGRs not able to be routed.

The Storage Management function takes as input the list of resources, the list of routed IGRs, the list of IGRs that are not able to be routed, and the maximum storage size for each resource. While not all of the resources have been considered, lines 2 through 26 are executed. Line 3 chooses a resource from those still to be considered. Lines 5 to 25 get executed for each time-step of the time horizon. In lines 6 to 8 , each IGR that has completely transited through the current resource by the time-step under consideration, gets removed from the resources' storage, from the time-step under consideration to the end of the time horizon. In lines 9 to 11 , each IGR that has been completely consumed by the current resource, by the time-step under consideration, gets removed from the resources' storage, from the time-step under consideration to the end of the time horizon. Now, if the storage for the current resource at the time-step under consideration is above the capacity, in lines 13 to 15 each IGR that was generated by the current resource and has been completely transited through the current resource (i.e., completely sent to the next resource along the path to the consumer resource) by the time-step under consideration gets removed from the resources' storage, from the time-step under consideration to the end of the time horizon. Up to this point, all IGRs that were to be routed (as determined from the Information Routing function) are still valid. Line 17 gets the list of IGRs that are scheduled to be generated by the current resource no later than the time-step under consideration, and line 18 sorts this list. While the storage for the current resource at the time-step under consideration is above the maximum allowed, an IGR from the sorted IGR list is chosen, and it is removed from routing. This continues until the storage constraints are met for the current resource at the time-step under consideration. Output from the Storage Management function is an updated list of IGRs chosen to be routed and IGRs not able to be routed. 


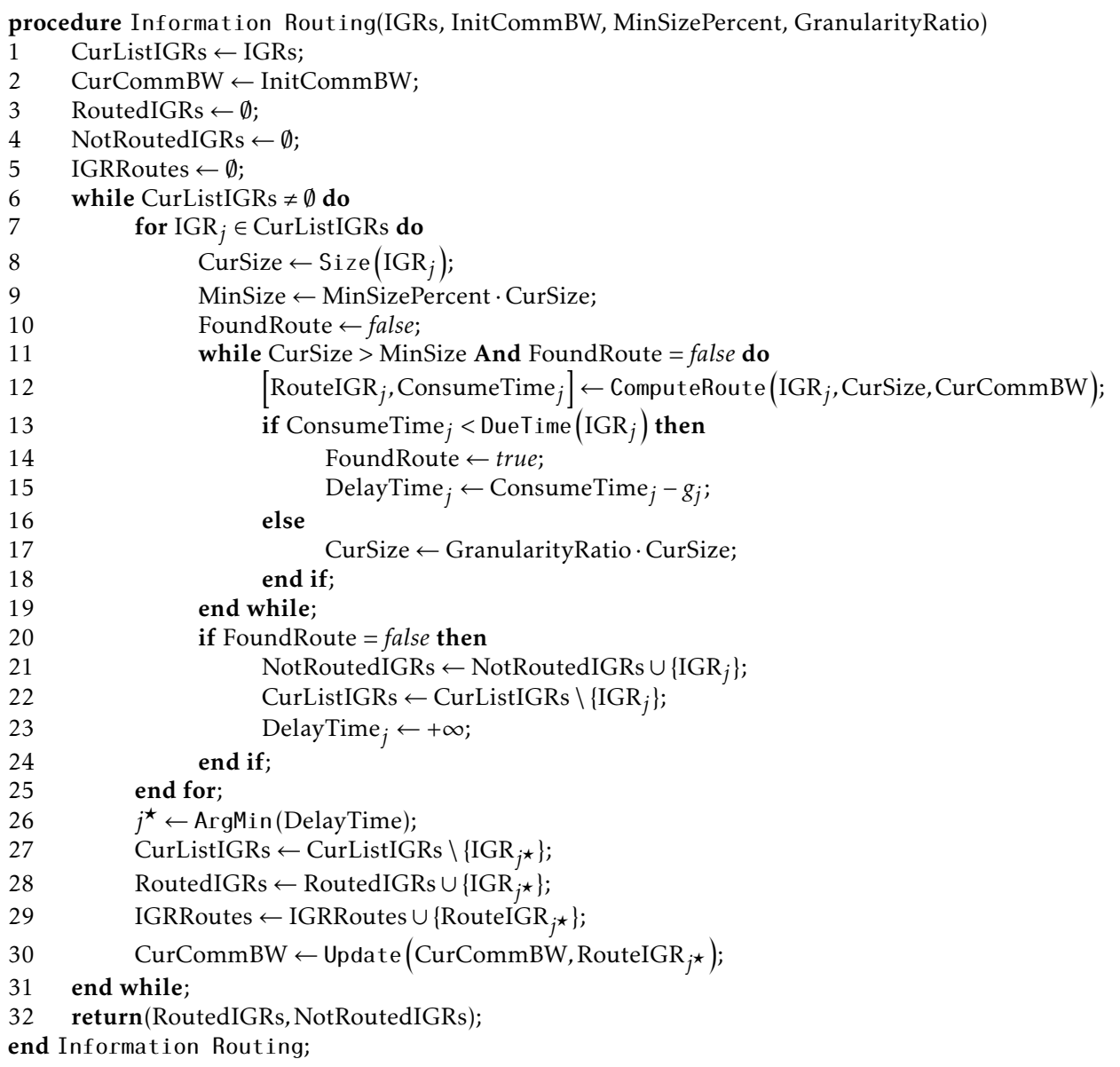

Figure 2: Pseudo-code for the Information Routing phase of the heuristic.

\section{Computational Experiments}

\subsection{Test Environment}

Java jdk 1.8.0_25 was used to code up the heuristic, the actual exact MILP, and the generation of scenario data. CPLEX Optimization Studio V12.6.3 [27] was called to solve the exact MILP. Matlab R2011b (7.13.0.564) [34] was used to generate combinations of input parameters, as well as to analyze the results of all experiments. All experiments were conducted on a Dell Precision Tower 7810 with an Intel(R) Xeon(R) CPU E5-2630v3@2.40GHz 2.40 GHz with 32.0 GB memory.

\subsection{Experimental Results}

The aim of our experiments were to investigate the solution quality found by the heuristic, as compared with commercial software, as well as to determine how close to optimal were the solutions found by the heuristic. We used the commercial software CPLEX [27] to solve the linearized formulation of the mathematical model presented in Section 2. However, as can be seen from the results that follow, for some of the scenarios CPLEX was not able to find solutions significantly close to optimal, within the time-limits to find a solution. Thus, we also ran CPLEX to solve the linearized formulation, providing CPLEX an initial solution found from running the heuristic. These two sets of experiments involving CPLEX are described as "CPLEX w/o Init. Soln." and "CPLEX w/ Init. Soln.", respectively. To test the heuristic, as well as CPLEX, we created numerous scenarios, varying the number of resources $(\mathrm{N})$ and the density of the underlying communication network topology, as displayed in Table 2 .

The maximum density of the underlying communication network topology is given by Equation (33), where $N$ is the set of nodes, or resources, of the network and $E$ is the set of communication links, or edges, between nodes of the network. So, a maximum density of 0.8 means that $20 \%$ of the edges have been deleted from the complete network. We note that deleting these edges has the same effect as enforcing that those edges have a maximum bandwidth of 0 over the timehorizon. Also it is important to point out that this gives the maximum density of the network over any given time. In actuality, based on temporal bandwidth parameters defined ('Bandwidth per Directed Edge per Resource' in Table 3, there exist the potential for two nodes to have 0 available direct bandwidth at a given time-step, even though this edge was not explicitly removed from the network. This can be seen as modeling the possibility for a node to be kinematically out of range of another, and hence not able to communicate, or for a node to be operating in 'radio silence' at that 
Table 3: Parameter categories and values used for the numerical experiments. For those parameters with only a minimum value, that is the fixed value for the parameter. For those parameters that have a minimum and maximum value, the distribution listed is the probability distribution through which a specific parameter is determined, via a random draw.

\begin{tabular}{lccc} 
Parameter & Min. Value & Max. Value & Dist. \\
\hline Number Time-Steps & 20 & & \\
\hline $\begin{array}{l}\text { Bandwidth per Directed Edge } \\
\text { per Resource }\end{array}$ & 0 & 100 & Uniform \\
\hline $\begin{array}{l}\text { Number of IGRs in Scenario } \\
\text { per Resource }\end{array}$ & 5 & 10 & Uniform \\
\hline $\begin{array}{l}\text { Time Delay Between IGR } \\
\text { Generation and Consumption }\end{array}$ & 7 & 20 & Uniform \\
\hline $\begin{array}{l}\text { Resources that Neither Generate } \\
\text { nor Consume IGRs }\end{array}$ & $0.2 \cdot N\rceil$ & & \\
\hline IGR Size & 25 & 250 & Uniform \\
\hline $\begin{array}{l}\text { Storage Capacity } \\
\text { per Resource }\end{array}$ & 750 & 1500 & Uniform \\
\hline Granularity Ratio & 0.9 & & \\
\hline MinSizePercent & 0.8 & & \\
\hline \hline
\end{tabular}

Table 4: Solution quality analysis, according to Equation (34), for the mathematical formulation using CPLEX, without and with an initial input solution.

\begin{tabular}{|c|c|c|c|c|c|c|}
\hline \multirow{3}{*}{$\begin{array}{c}\text { Num. } \\
\text { Resources }\end{array}$} & \multirow[b]{3}{*}{ Density } & \multicolumn{3}{|c|}{ CPLEX w/o Init. Soln. } & \multicolumn{2}{|c|}{ CPLEX w/ Init. Soln. } \\
\hline & & \% Time Opt. & Mean & $\%$ Time Feasible & \% Time Opt. & Mean \\
\hline & & Soln. Found & Soln. GAP & Soln. Not Found & Soln. Found & Soln. GAP \\
\hline 3 & 1 & 80 & 0.0012 & 0 & 80 & 0.0012 \\
\hline 3 & 0.67 & 100 & & 0 & 100 & \\
\hline 3 & 0.33 & 100 & & 0 & 100 & \\
\hline 4 & 1 & 100 & & 0 & 100 & \\
\hline 4 & 0.67 & 90 & 0.0013 & 0 & 90 & 0.0012 \\
\hline 4 & 0.33 & 90 & 0.0001 & 0 & 90 & 0.0001 \\
\hline 5 & 1 & 80 & 0.0002 & 0 & 90 & 0.0001 \\
\hline 5 & 0.67 & 60 & 0.0029 & 0 & 50 & 0.0027 \\
\hline 5 & 0.33 & 70 & 0.0006 & 0 & 70 & 0.0006 \\
\hline 6 & 1 & 60 & 0.0207 & 0 & 50 & 0.0035 \\
\hline 6 & 0.67 & 60 & 0.0022 & 0 & 70 & 0.0093 \\
\hline 6 & 0.33 & 100 & & 0 & 100 & \\
\hline 8 & 1 & 0 & 0.0343 & 0 & 0 & 0.0133 \\
\hline 8 & 0.67 & 0 & 0.0775 & 0 & 10 & 0.0368 \\
\hline 8 & 0.33 & 20 & 0.0109 & 0 & 20 & 0.0115 \\
\hline 10 & 1 & 0 & 0.1149 & 0 & 0 & 0.0274 \\
\hline 10 & 0.67 & 0 & 0.1031 & 0 & 0 & 0.0240 \\
\hline 10 & 0.33 & 0 & 0.0636 & 0 & 0 & 0.0890 \\
\hline 12 & 1 & 0 & 0.2145 & 0 & 0 & 0.0390 \\
\hline 12 & 0.67 & 0 & 0.1308 & 0 & 0 & 0.0349 \\
\hline 12 & 0.33 & 0 & 0.3658 & 0 & 0 & 0.2128 \\
\hline 15 & 1 & 0 & 0.4977 & 0 & 0 & 0.0678 \\
\hline 15 & 0.67 & 0 & 0.2130 & 0 & 0 & 0.0637 \\
\hline 15 & 0.33 & 0 & 0.4390 & 10 & 0 & 0.1810 \\
\hline 20 & 1 & 0 & 0.9583 & 90 & 0 & 0.2988 \\
\hline 20 & 0.67 & 0 & 0.6205 & 30 & 0 & 0.1122 \\
\hline 20 & 0.33 & 0 & 0.2709 & 10 & 0 & 0.2507 \\
\hline 25 & 1 & 0 & 1.0000 & 100 & 0 & 0.2614 \\
\hline 25 & 0.67 & 0 & 1.0000 & 100 & 0 & 0.3739 \\
\hline 25 & 0.33 & 0 & 0.3702 & 10 & 0 & 0.2155 \\
\hline
\end{tabular}

time-step, due to external environmental conditions model a node failure [35, 36, 37] if a particular node (e.g., weather, topography, etc). In addition, this can has 0 available bandwidth at a given time over all edges 
Table 5: Average normalized distance, computed according to Equation (35), between the heuristic and CPLEX with/without the initial solution, for those cases where CPLEX found at least a feasible solution. Note: An entry is left blank whenever CPLEX was not able to find a feasible solution for any of the scenarios.

\begin{tabular}{cc|cc}
\hline $\begin{array}{c}\text { Num. } \\
\text { Resources }\end{array}$ & Density & $\begin{array}{c}\text { CPLEX w/out } \\
\text { Init Soln }\end{array}$ & $\begin{array}{c}\text { CPLEX w/ } \\
\text { Init Soln }\end{array}$ \\
\hline 3 & 1 & -0.132 & -0.132 \\
3 & 0.67 & -0.127 & -0.127 \\
3 & 0.33 & -0.115 & -0.115 \\
4 & 1 & -0.072 & -0.072 \\
4 & 0.67 & -0.174 & -0.174 \\
4 & 0.33 & -0.330 & -0.330 \\
5 & 1 & -0.098 & -0.098 \\
5 & 0.67 & -0.194 & -0.194 \\
5 & 0.33 & -0.212 & -0.212 \\
6 & 1 & -0.071 & -0.089 \\
6 & 0.67 & -0.151 & -0.145 \\
6 & 0.33 & -0.481 & -0.481 \\
8 & 1 & -0.064 & -0.085 \\
8 & 0.67 & -0.079 & -0.128 \\
8 & 0.33 & -0.400 & -0.400 \\
10 & 1 & 0.024 & -0.071 \\
10 & 0.67 & -0.013 & -0.120 \\
10 & 0.33 & -0.281 & -0.254 \\
12 & 1 & 0.158 & -0.066 \\
12 & 0.67 & 0.070 & -0.100 \\
12 & 0.33 & 0.300 & -0.102 \\
15 & 1 & 0.779 & -0.051 \\
15 & 0.67 & 0.090 & -0.086 \\
15 & 0.33 & 0.491 & -0.114 \\
20 & 1 & 1.186 & -0.037 \\
20 & 0.67 & 0.536 & -0.066 \\
20 & 0.33 & -0.068 & -0.108 \\
25 & 1 & & -0.035 \\
25 & 0.67 & & -0.036 \\
25 & 0.33 & 0.109 & -0.104 \\
\hline & & &
\end{tabular}

connecting it to other nodes.

$$
\text { Density }=\frac{|E|}{|N| \cdot(|N|-1)}
$$

For each combination of number of resources and density, 10 Monte-Carlo scenarios were created randomly. For each scenario generated, the remaining parameters were derived from the information in Table 3 , and then kept fixed for the scenario. In addition, the coefficients of the two terms in the objective function (time-delay and size), $\omega_{d}$ and $\omega_{s}$, were fixed to 0.7 and 0.3 for all scenarios. Each approach ("CPLEX w/o Init. Soln.", "CPLEX w/ Init. Soln.", and the heuristic) were given 900 seconds to solve each scenario instance. For those cases where CPLEX did not solve the problem to optimality within the 900 seconds, the best solution found by CPLEX is considered. We note that there are cases in the tables to follow where CPLEX was not able to find even a feasible solution by the time-limit, hinting at the limitations of commercial software as the size of the problems get large.

Table 4 examines the results just from the exact approach, using CPLEX (without and with an initial solution). For each combination of number of resources and communication network density, columns 3-5 deal with the solution found by CPLEX without an initial solution, while columns 6-7 are concerned with the solution found by CPLEX with an initial solution provided. Columns 3 and 6 show that as the problem size gets larger, CPLEX has a more difficult time in finding the optimal solution. And for most of the problem sizes, in terms of providing an optimal solution, there is no difference between CPLEX without or with an initial solution. Column 5 shows the percentage of times CPLEX without an initial solution is unable to find a feasible solution to the problem. There is always the trivial feasible solution where no IGRs are routed, so CPLEX unable to find a feasible solution means that CPLEX ran out of memory in the creation of the mathematical model. Again this is as expected; as the problems get larger, more variables and constraints are needed, and eventually memory issues are encountered. Columns 4 and 7 show the mean gap between the upper bound on the solution value computed in CPLEX and the best solution found by CPLEX. For each scenario, this gap is computed as in Equation 
Table 6: Mean Time Delay between generation and consumption of IGRs chosen to be routed, for each solution approach. For those cases where CPLEX is not able to find a feasible solution within the time-limit for any scenarios, the entry is left blank. The results are shown as mean:standard deviation.

\begin{tabular}{|c|c|c|c|c|}
\hline $\begin{array}{c}\text { Num. } \\
\text { Resources }\end{array}$ & Density & $\begin{array}{r}\text { CPLEX w/out } \\
\text { Init Soln }\end{array}$ & Heuristic & $\begin{array}{r}\text { CPLEX w/ } \\
\text { Init Soln }\end{array}$ \\
\hline 3 & 1 & $8.956: 0.758$ & 5.070:1.120 & $8.937: 0.738$ \\
\hline 3 & 0.67 & $9.691: 1.387$ & 4.968:0.897 & 9.658:1.389 \\
\hline 3 & 0.33 & 10.223:0.993 & $4.651: 0.722$ & 10.194:0.960 \\
\hline 4 & 1 & $9.268: 0.954$ & $4.105: 0.335$ & $9.278: 0.952$ \\
\hline 4 & 0.67 & $9.867: 0.734$ & 5.059:0.680 & 9.856:0.697 \\
\hline 4 & 0.33 & 10.096:1.153 & $6.036: 1.208$ & $10.128: 1.168$ \\
\hline 5 & 1 & 9.004:0.676 & $3.773: 0.327$ & $9.030: 0.663$ \\
\hline 5 & 0.67 & $9.762: 0.779$ & 5.073:0.433 & 9.735:0.756 \\
\hline 5 & 0.33 & $9.588: 1.137$ & $5.321: 0.700$ & $9.595: 1.128$ \\
\hline 6 & 1 & 9.300:0.571 & $4.021: 0.296$ & $9.270: 0.582$ \\
\hline 6 & 0.67 & 9.502:0.988 & 5.257:0.345 & $9.571: 0.953$ \\
\hline 6 & 0.33 & 9.925:1.093 & $6.361: 0.861$ & $9.944: 1.050$ \\
\hline 8 & 1 & $9.201: 0.700$ & $4.016: 0.247$ & 9.186:0.639 \\
\hline 8 & 0.67 & 9.233:0.558 & 4.704:0.340 & 9.426:0.830 \\
\hline 8 & 0.33 & 10.047:0.665 & $6.433: 0.560$ & 10.099:0.667 \\
\hline 10 & 1 & $9.010: 0.560$ & $3.807: 0.321$ & $9.248: 0.613$ \\
\hline 10 & 0.67 & $8.504: 0.580$ & $4.517: 0.316$ & 8.788:0.658 \\
\hline 10 & 0.33 & $9.460: 0.457$ & $6.117: 0.519$ & 9.928:0.984 \\
\hline 12 & 1 & 8.632:0.482 & $3.846: 0.234$ & 9.242:0.494 \\
\hline 12 & 0.67 & $8.950: 0.458$ & $4.684: 0.313$ & $9.324: 0.497$ \\
\hline 12 & 0.33 & $9.362: 0.603$ & $6.194: 0.334$ & $11.301: 0.979$ \\
\hline 15 & 1 & 7.573:0.398 & $3.820: 0.122$ & 9.599:0.288 \\
\hline 15 & 0.67 & $8.590: 0.698$ & $4.411: 0.204$ & $9.197: 0.570$ \\
\hline 15 & 0.33 & 9.449:1.149 & 5.924:0.569 & 11.035:0.874 \\
\hline 20 & 1 & 7.633:0.000 & $3.688: 0.148$ & 9.692:0.348 \\
\hline 20 & 0.67 & $7.586: 0.312$ & $4.376: 0.068$ & $9.961: 0.541$ \\
\hline 20 & 0.33 & $8.684: 0.512$ & $5.866: 0.269$ & $10.534: 1.066$ \\
\hline 25 & 1 & & $3.721: 0.096$ & $9.629: 0.312$ \\
\hline 25 & 0.67 & & $4.366: 0.211$ & 10.469:0.287 \\
\hline 25 & 0.33 & $8.471: 0.424$ & 5.537:0.139 & 10.359:1.046 \\
\hline
\end{tabular}

(34), where $u b$ is the upper bound on the solution value and $s f$ is the solution found by CPLEX. We note that when CPLEX finds the optimal solution, $u b=s f$ and the GAP is therefore 0 , and when CPLEX is not able to find even a feasible solution, we assigned the trivial solution of $s f=0$ (the worst feasible solution for the model), resulting in a GAP value of 1 .

$$
G A P=\frac{u b-s f}{u b}
$$

What is apparent from Columns 4 and 7 of Table 4 is that CPLEX with an initial solution performs better most of the time as compared to CPLEX without an initial solution. In most cases, there is a significant decrease in the mean gap. However, there are a few cases that are counter-intuitive. Specifically when the number of resources is 6,8 , or 10 and the densities are $0.67,0.33$, or 0.33 respectively. For these three combinations, CPLEX without an initial solution has a smaller mean gap than does CPLEX with an initial solution. This is because there was one scenario in each of these combinations where the heuristic found a solution not close to the optimal, and CPLEX had a difficult time given the heuristic solution as the initial solution, i.e., the heuristic found a local maximum, and CPLEX had a difficult time finding a solution better than this local maximum.

Table 5 shows the average normalized distances between the heuristic solutions and the CPLEX solutions without and with the initial solution. Each normalized distance is computed according to Equation (35), where $\left\{x_{j}^{h}, s_{j}^{h}\right\}$ is the solution found by the heuristic, $\left\{x_{j}^{c}, s_{j}^{c}\right\}$ is the solution to the linearized formulation using CPLEX, and $f$ is the objective function. Since we have a maximization problem, values of Equation (35) larger than 0 indicate that the heuristic is finding a better solution than is CPLEX, while those less than 0 indicate an heuristic solution worse than that found by CPLEX. First off, as expected, all of the values in the last column of Table 5 are negative, because the worst solution 'CPLEX with initial solution' can find is the same solution as found by the heuristic since this is the solution provided as input to CPLEX. For less than 12 resources, CPLEX without an initial solution 
Table 7: Average runtime (in seconds) for each solution approach to find a solution. For those cases where CPLEX is not able to find a feasible solution within the time-limit, the returned time by CPLEX is still used in this computation.

\begin{tabular}{|c|c|c|c|c|}
\hline $\begin{array}{c}\text { Num. } \\
\text { Resources }\end{array}$ & Density & $\begin{array}{l}\text { CPLEX w/out } \\
\text { Init Soln }\end{array}$ & Heuristic & $\begin{array}{l}\text { CPLEX w/ } \\
\text { Init Soln }\end{array}$ \\
\hline 3 & 1 & 218.705 & 0.002 & 224.092 \\
\hline 3 & 0.67 & 48.794 & 0.000 & 132.075 \\
\hline 3 & 0.33 & 10.150 & 0.000 & 12.552 \\
\hline 4 & 1 & 22.205 & 0.003 & 30.301 \\
\hline 4 & 0.67 & 179.300 & 0.000 & 190.852 \\
\hline 4 & 0.33 & 123.936 & 0.001 & 155.227 \\
\hline 5 & 1 & 388.937 & 0.000 & 337.506 \\
\hline 5 & 0.67 & 433.410 & 0.002 & 498.094 \\
\hline 5 & 0.33 & 465.390 & 0.001 & 444.524 \\
\hline 6 & 1 & 469.135 & 0.002 & 634.885 \\
\hline 6 & 0.67 & 595.497 & 0.002 & 502.483 \\
\hline 6 & 0.33 & 109.626 & 0.000 & 161.185 \\
\hline 8 & 1 & 901.857 & 0.008 & 901.757 \\
\hline 8 & 0.67 & 901.863 & 0.006 & 865.321 \\
\hline 8 & 0.33 & 822.245 & 0.005 & 761.825 \\
\hline 10 & 1 & 904.283 & 0.006 & 903.338 \\
\hline 10 & 0.67 & 905.129 & 0.005 & 905.198 \\
\hline 10 & 0.33 & 903.219 & 0.002 & 903.443 \\
\hline 12 & 1 & 904.817 & 0.011 & 905.141 \\
\hline 12 & 0.67 & 905.752 & 0.003 & 906.519 \\
\hline 12 & 0.33 & 905.980 & 0.003 & 904.835 \\
\hline 15 & 1 & 910.124 & 0.044 & 910.081 \\
\hline 15 & 0.67 & 1310.217 & 0.005 & 1071.725 \\
\hline 15 & 0.33 & 1161.690 & 0.005 & 1124.190 \\
\hline 20 & 1 & 2872.113 & 0.083 & 2066.906 \\
\hline 20 & 0.67 & 1283.765 & 0.009 & 994.237 \\
\hline 20 & 0.33 & 958.069 & 0.005 & 1153.269 \\
\hline 25 & 1 & 4612.964 & 0.128 & 2845.093 \\
\hline 25 & 0.67 & 6458.231 & 0.006 & 6317.989 \\
\hline 25 & 0.33 & 1672.833 & 0.006 & 1980.635 \\
\hline
\end{tabular}

is also able to find a better solution on average than is the heuristic. However, after this point, for almost all cases the heuristic is finding a better solution than is CPLEX without an initial solution. Again, this is to be expected, because as the size of the problem grows, commercial software will have more difficulty in solving the larger problems and the heuristic will begin to find better solutions. But in comparing the heuristic with CPLEX with an initial solution, the average normalized distance between solutions is never that large on average, showing that the heuristic is able to find a good-quality solution as compared with commercial software.

$$
\frac{f\left(x_{j}^{h}, s_{j}^{h}\right)-f\left(x_{j}^{c}, s_{j}^{c}\right)}{f\left(x_{j}^{c}, s_{j}^{c}\right)}
$$

Table 6 presents, for those IGRs chosen to be routed, the mean time delay between IGR generation and IGR consumption. Column 3 of Table 6 shows the mean delay for the CPLEX solution when no initial solution is provided, Column 4 shows the mean delay for the heuristic solution, while Column 5 shows the mean delay for the CPLEX solution when an initial solution is provided. As is clear from this table, for those IGRs routed, the heuristic is able to route them much quicker than is CPLEX. This is regardless of the size of the problem. The rationale for this is that the heuristic is choosing IGRs to route in a greedy way, while CPLEX considers the routing of all IGRs simultanously.

Table 7 presents the average runtime for each of the approaches, in seconds. As is clear, the developed heuristic is quite fast in finding solutions for all of the scenario classes. It is also clear that the heuristic takes longer to run on average when the communication network topology is dense as opposed to sparse. Both of the CPLEX approaches take much longer than does the heuristic. Even when the number of resources is as small as 8 , these approaches reach (or almost reach) the time limit of 900 seconds. Hence, to get sightly better solutions (according to the objective function) CPLEX needs much more time than does the heuristic. We note that while CPLEX was given a time limit of 900 seconds, there were cases where CPLEX does take 
longer to complete. This is due to CPLEX not being able to 'halt' processing exactly when the time limit is reached.

\section{Conclusions and Future Re- search}

In this research, we have examined the problem of routing information amongst a set of resources, when there exists a dynamic communication network topology with limited, time-varying, bandwidth, over a given time horizon. A rigorous mathematical formulation was developed to model the problem, with the objective being to minimize the time delay of the information that can be routed while at the same time maximizing the size of the information routed. This formulation is nonlinear, but a linearized version can be derived through standard techniques of operations research. As the problem is NP-hard, a heuristic has been developed to efficiently find good-quality solutions. Numerous Monte-Carlo simulations were performed on problems with varied resources and communication network topology density. As can be seen in the results, for smaller sized problems commercial software is able to find a better solution on average than is the heuristic. And when the commercial software uses as input the solution found by the heuristic, even better results are obtained. As the size of the problem increases, however, the heuristic begins finding much better solutions compared with the commercial software. And even when the commercial software is provided the heuristic solution as input, the commercial software is not able to improves significantly on this solution. Looking at the metric of the actual timedelay for those IGRs chosen to be routed, it is clear that the heuristic is better able to route those chosen IGRs. When coupled with the time needed for the heuristic to find a good quality solution versus the commercial software, it is clear that the heuristic is outperforming the commercial software.

Future research includes looking at specific network topology structures from realistic military and civil applications, considering the related problem of finding the minimum network temporal connectivity necessary to ensure certain information is able to be routed within time bounds, as well as considering the problem from a decentralized control paradigm, when no resource has knowledge about the network as a whole, but rather must consider only local network knowledge and make independent routing decision. In addition, incorporating the concept of network packet errors will provide added complexity and realism to the problem and scenarios considered.

Conflict of Interest The authors declare no conflict of interest.

Acknowledgment This research was Supported by Office of Naval Research Project N00014-15-C-5163.

\section{References}

[1] M. J. Hirsch, A. Sadeghnejad, and H. Ortiz-Pena, "Shortest paths for routing information over temporally dynamic communication networks," in Proceedings of the IEEE Military Communications Conference, pp. 587-591, 2017.

[2] I. Akyildiz, W. Su, Y. Sankarasubramaniam, and E. Cayirci, "Wireless sensor networks: A survey," Computer Networks, vol. 38, pp. 393-422, 2002.

[3] J. Al-Karaki, , and A. Kamal, "Efficient virtual-backbone routing in mobile ad hoc networks," in Elsevier Computer Networks, vol. 52, pp. 327-350, 2008.

[4] E. Huang, W. Hu, J. Crowcroft, and I. Wassell, "Towards commercial mobile ad hoc network applications: a radio dispatch system," 6th ACM International Symposium on Mobile Ad Hoc Networking and Computing, pp. 355-365, 2005.

[5] “Army 18.1 Small Business Innovation Research (SBIR) Proposal Topic 025: An Adaptively Covert, High Capacity RF Communications / Control Link,” tech. rep., 2018.

[6] “Air Force 18.1 Small Business Innovation Research (SBIR) Proposal Topic 019: Novel Battle Damage Assessment Using Snesor Networks," tech. rep., 2018.

[7] C. Howard, "UAV command, control, and communication," Military and Aerospace Electronics, vol. 24, 2013.

[8] B. Vignac, F. Vanderbeck, and B. Jaumard, "Reformulation and decomposition approached for traffic routing in optical networks," Networks, vol. 67, pp. 277-298, 2016.

[9] J. Brodkin, “Tropical storm Harvey takes out 911 centers, cell towers, and cable networks," ars Technica, August 282017.

[10] L. K. Comfort and T. W. Haase, "Communication, choerence, and collective action: The impage of Hurrican Katrina on the communication infrastructure," Public Works Management $\mathcal{E}$ Policy, vol. 11, no. 1, pp. 1-16, 2006.

[11] U.S. Air Force, “USAF Strategic Master Plan,” tech. rep., 2015.

[12] “Air Force 17.1 Small Business Innovation Research (SBIR) Proposal Topic 047: Resilient Communication for Contested Autonomous Manned/Unmanned Teaming," tech. rep., 2017.

[13] D. L. Hall and J. Llinas, "An introduction to multisensor data fusion," in Proceedings of the IEEE, vol. 85-1, pp. 6-23, 1997.

[14] E. Bosse, J. Roy, and S. Wark, eds., Concepts, Models, and Tools for Information Fusion. Artech House, 2007.

[15] F. A. A. U.S. Department of Transportation, "Aviation Maintenance Technician Handbook - Airframe, Volume 2," tech. rep., 2012.

[16] "Navy 17.A Small Business Technology Transfer (STTR) Proposal Topic T029: Multi-Vehicle Collaboration with Minimal Communication and Minimal Energy," tech. rep., 2017.

[17] I. Jebadurai, E. Rajsingh, and G. Paulraj, "Enhanced dynamic source routing protocol for detection and prevention of sinkhole attack in mobile ad hoc networks," International Journal of Network Science, vol. 1, pp. 63-79, 2016.

[18] U. Paul, M. Buddhikot, and S. Das, "Opportunistic traffic scheduling in cellular data networks," IEEE International Symposium on Dynamic Spectrum Access Networks, pp. 339-349, 2012.

[19] J. Al-Karaki, R. Ul-Mustafa, and A. Kamal, "Data aggregation and routing in wireless sensor networks: Optimal and heuristic algorithms," in Elsevier Computer Networks, vol. 53, pp. 945-960, 2009.

[20] M. J. Hirsch, H. Ortiz-Pena, R. Nagi, A. Stotz, and M. Sudit, "On the optimization of information workflow," in Dynamics of Information Systems: Mathematical Foundations (A. Sorokin, R. Murphey, M. Thai, and P. Pardalos, eds.), vol. 20, pp. 43-65, Springer, 2012. 
[21] M. J. Hirsch and H. Ortiz-Pena, "Information supply chain optimization with bandwidth limitations," International Transactions in Operational Research, vol. 24, no. 5, pp. 993-1022, 2017.

[22] E. Grotli and T. Johansen, "Path and data transmission planning for cooperating uavs in delay tolerant network," in Globecom Workshop, pp. 1568-1573, IEEE, 2012.

[23] S. Basagni, C. Petrioli, R. Petroccia, and M. Stojanovic, "Optimizing network performance through packet fragmentation in multi-hop underwater communications," in OCEANS, pp. 1-7, IEEE, 2010.

[24] P. Kansal and A. Bose, "Bandwidth and latency requirements for smart transmission grid applications," IEEE Transactions on Smart Grid, vol. 3, pp. 1344-1352, 2012.

[25] M. Greenberg, Advanced Engineering Mathematics. Pearson, 2nd ed., 1998.

[26] L. Wolsey, Integer Programming. Wiley, 1998.

[27] I. CPLEX, "http://www-01.ibm.com/software/commerce /optimization/cplex-optimizer/," Accessed January 2017.

[28] LINDO, “http://www.lindo.com/," Accessed July 2016.

[29] J. Lenstra and A. R. Kan, "Complexity of vehicle and scheduling problems," Networks, vol. 11, pp. 221-227, 1981.
[30] M. Solomon and J. Desrosiers, "Time window constrained routing and scheduling problem," Transportation Science, vol. 22, pp. $1-13,1988$.

[31] M. Dror and P. Trudeau, "Split delivery routing," Naval Research Logistics, vol. 37, pp. 383-402, 1990.

[32] G. Ausiello, M. Protasi, A. Marchetti-Spaccamela, G. Gambosi, P. Crescenzi, and V. Kann, Complexity and Approximation: Combinatorial Optimization Problems and Their Approximability Properties. Springer-Verlag New York, Inc., 1st ed., 1999.

[33] M. R. Garey and D. S. Johnson, Computers and Intractability: A Guide to the Theory of NP-Completeness. New York, NY, USA: W. H. Freeman \& Co., 1979.

[34] Matlab, "http://www.mathworks.com/products/matlab/," Accessed January 2017.

[35] B. Szymanski, X. Lin, A. Asztalos, and S. Sreenivasan, "Failure dynamics of the global risk network," Scientific Reports, vol. 5, pp. 1-14, 2015.

[36] R. Liu, M. Li, and C. Jia, "Cascading failures in coupled networks: The critical role of node-coupling strength across networks," Scientific Reports, vol. 6, pp. 1-6, 2016.

[37] K. Kim, J. Jin, and S. Jin, "Classification between failed nodes and left nodes in mobile asset tracking systems," Sensors, vol. 16, pp. 1-18, 2016. 